\title{
Transverse-to-Longitudinal Phase Space Exchange: a Versatile Tool for Shaping the Current and Energy Profiles of Relativistic Electron Bunches
}

\author{
P. Piot ${ }^{* \dagger}$, Y.-E Sun ${ }^{\dagger}$, A. S. Johnson**, A. H. Lumpkin**, T. Maxwell*,†, J. Ruan** \\ and R. Thurman-Keup** \\ ${ }^{*}$ Northern Illinois Center for Accelerator \& Detector Development and Department of Physics, \\ Northern Illinois University, DeKalb IL 60115, USA \\ ${ }^{\dagger}$ Accelerator Physics Center, Fermi National Accelerator Laboratory, Batavia, IL 60510, USA \\ ${ }^{* *}$ Accelerator Division, Fermi National Accelerator Laboratory, Batavia, IL 60510, USA
}

\begin{abstract}
Over the recent years, the emergence of accelerator beamlines capable of exchanging the phase space coordinates between two degrees of freedom have opened the path toward the precise control of phase space distribution and in particular to the production of relativistic electron beams with shaped current profiles. After briefly reviewing the technique, we present its application to produce a train of sub-picosecond microbunches and report on its experimental implementation at the Fermilab's A0 photoinjector facility.
\end{abstract}

Keywords: beam dynamics, electron beams, phase-space manipulations, beam shaping PACS: 29.27.-a, 41.85.-p, 41.75.F

\section{INTRODUCTION}

Recent advanced applications of electron-beam accelerators have increased the demand for precise phase-space control schemes. In particular, electron bunches with a well-defined temporal distribution are often desired. An interesting class of temporal distributions consists of a train of microbunches with sub-picosecond duration and separation. Applications of such trains of microbunches include the generation of super-radiant radiation [1] or the resonant excitation of wakefields in plasma wakefield accelerators or dielectric-loaded structures [2]. To date there are very few techniques capable of reliably providing this class of beam [3]. We have recently explored an alternative technique based on the use of a transverse-to-longitudinal phase space exchange method $[4,5]$. The method consists of shaping the beam's transverse density to produce the desired horizontal profile. The horizontal profile is then mapped onto the longitudinal profile by a beamline capable of exchanging the phase space coordinates between the horizontal and longitudinal degrees of freedom. Therefore the production of a train of microbunches simply relies on generating a set of horizontally-separated beamlets upstream of the beamline. The backbone of the proposed technique is the transverse-to-longitudinal phase space exchange which was recently proposed as a means to mitigate the microbunching instability in high-brightness electron beams [6] or to improve the performance of single-pass FELs [7]. A simple optical lattice capable of performing this phase space exchange consists of a horizontally-deflecting cavity, operating on the $\mathrm{TM}_{110}$ mode, flanked by two horizontally-dispersive sections henceforth referred to as "doglegs" [8]. Under the thin lens approximation the initial transverse phase space coordinates $\left(x_{0}, x_{0}^{\prime}\right)$ are mapped to the longitudinal phase space coordinates $(z, \delta)$ following [9]

$$
\left\{\begin{array}{l}
z=-\frac{\xi}{\eta} x_{0}-\frac{L \xi-\eta^{2}}{\eta} x_{0}^{\prime} \\
\delta=-\frac{1}{\eta} x_{0}-\frac{L}{\eta} x_{0}^{\prime}
\end{array}\right.
$$

where $L$ is the distance between the dogleg's dipoles, and $\eta$ and $\xi$ are respectively the horizontal and longitudinal dispersions generated by one dogleg. Besides swapping the horizontal and longitudinal emittances [10, 11], the coupling described by Eq. 1 can also be use of to arbitrarily shape the temporal distribution of an electron beam [12]. 


\section{EXPERIMENTAL GENERATION OF A TRAIN OF MICROBUNCHES}

The experiment was carried out at the Fermilab's A0 Photoinjector [13]; see Fig. 1. The electron bunches are generated via photoemission from a cesium telluride photocathode located on the back plate of a $1+1 / 2$ cell radiofrequency (rf) cavity operating at $1.3 \mathrm{GHz}$ (the "rf gun"). The rf gun is surrounded by three solenoidal lenses that control the beam's transverse size and emittance. The beam is then accelerated in a $1.3 \mathrm{GHz}$ superconducting TESLA rf cavity (the booster cavity) to a maximum energy of $16 \mathrm{MeV}$. Downstream of the booster cavity, the beam line includes a set of quadrupole and steering-dipole magnets along with an extensive diagnostics suite. The beamline then splits into two parallel lines.

The "straight ahead" beamline incorporates an horizontally-bending spectrometer equipped with a Cerium-dopped yttrium aluminum garnet (Cr:YAG) powder screen (labeled XS3 in Fig. 1) for energy measurement. The horizontal dispersion value at XS3 location is $\left|\eta_{x, X S 3}\right|=317 \mathrm{~mm}$.

The other beamline, the emittance exchange (EEX) beamline, implements the aforedescribed double-dogleg emittance exchanger [14]. The doglegs consist of dipole magnets with $\pm 22.5^{\circ}$ bending angles and each generate a horizontal and longitudinal dispersions of $\eta \simeq-33 \mathrm{~cm}$ and $\xi \simeq-12 \mathrm{~cm}$, respectively ${ }^{1}$. The deflecting cavity is a liquidNitrogen-cooled, normal-conducting, five-cell cavity operating on the $\mathrm{TM}_{110}$-like $\pi$-mode at $3.9 \mathrm{GHz}$ [15]. The lattice downstream of the EEX beamline includes three quadrupoles, a suite of standard beam diagnostics and a vertical spectrometer. At X24, the coherent transition radiation (CTR) produced as the beam impinges an Aluminum mirror is detected, and its analysis provides information on the bunch's temporal distribution. The vertical dispersion generated by the spectrometer at the XS4 Cr:YAG screen is $\left|\eta_{y, X S 4}\right|=944 \mathrm{~mm}$.

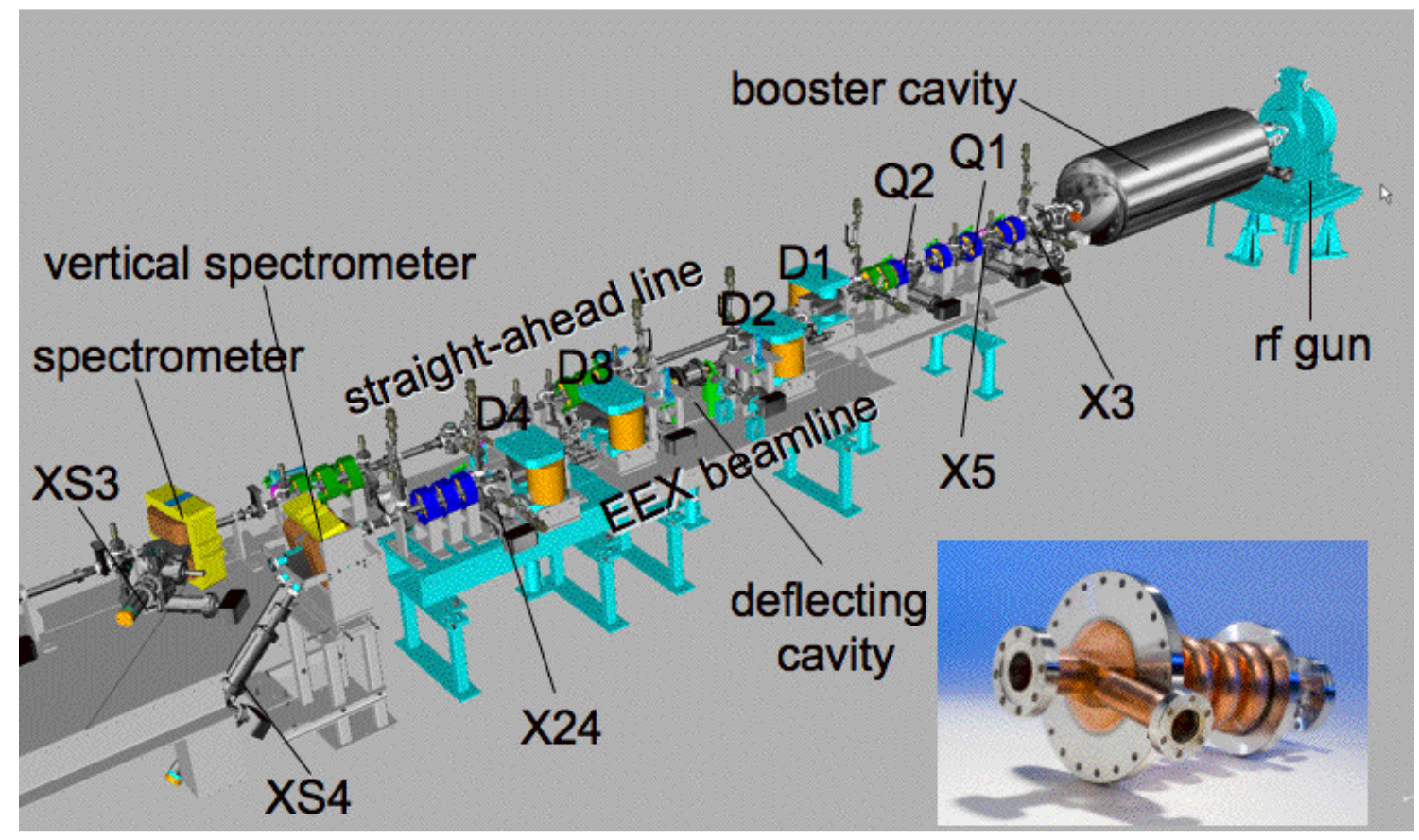

FIGURE 1. Three dimensional rendition of the A0 photoinjector showing the EEX beamline configuration. The labels Q $i$, D $i$ and $\mathrm{X} i$ respectively correspond to quadrupole and dipole magnets and $\mathrm{Cr}$-doped YAG screens. Only elements pertinent to the present experiment are labeled. The inset shows the deflecting cavity. [3D drawing courtesy of B. Popper, FNAL]

For the proof-of-principle experiment, the production of a transverse modulation was achieved by passing the 350$\mathrm{pC}$ beam through a set of remotely-controlled insertable vertical slits at X3. This multislit mask, nominally designed for single-shot transverse emittance measurements, consists of 50- $\mu \mathrm{m}$-wide slits made out of a 3-mm-thick tungsten plate. The slits are separated by $1 \mathrm{~mm}$. Less than $5 \%$ of the incoming beam is transmitted through the mask. This mask is not optimized for the present experiment: a smaller slits spacing would be more beneficial to increase the

\footnotetext{
${ }^{1}$ In our convention the head of the bunch is for $z<0$.
} 
total transmitted charge and to get a smaller separation between the bunches after the EEX beamline. The cylindricalsymmetric version of the particle-in-cell program ASTRA was used to model the beam dynamics from the photocathode to X3 [16]. Downstream of the X3 multislit mask, the produced beamlets are emittance-dominated and space charge effects do not significantly affect the beam dynamics. Therefore, the single-particle tracking program ELEGANT [17] was used to gain some insight in the experiment. In ELEGANT the deflecting cavity was modeled by the first-order thick-lens transfer matrix associated to five uncoupled $\mathrm{TM}_{110}$ pillbox cavities.

\section{Energy Modulations}

The beam was first diagnosed in the straight-ahead line and associated spectrometer to characterize the transverse modulation and ensure that no energy modulation was present and then transported through the EEX beamline with the deflecting cavity turned off. The horizontal modulation was still observable at X24 but no energy modulation could be seen at XS4; see Fig. 2 (a) and (b). Powering the cavity to its nominal deflecting voltage $\left(V_{x} \simeq 720 \mathrm{kV}\right)$ resulted in the suppression of the transverse modulation at X24 and the appearance of an energy modulation at XS4; see Fig. 2 (c) and (d). These observations are consistent with ELEGANT simulations and clearly demonstrate the ability of the EEX beamline to convert an incoming transverse density modulation into an energy modulation. For the present measurements the horizontal Courant-Snyder (CS) parameters upstream of the EEX beamline were empirically adjusted using quadrupoles $Q_{1}$ and $Q_{2}$ to maximize the energy modulation. The beamlets tilt seen at XS4 location when the cavity is turned off [see Fig. 2 (b) and corresponding simulation (f)] is due to the large horizontal dispersion. Using the settings of the three quadrupoles downstream of the EEX beamline, we computed the generated horizontal dispersion, with the deflecting cavity turned off, to be $\left|\eta_{x, X S 4}\right| \simeq 1.1 \mathrm{~m}$. The latter value results in an apparent $x-y$ coupling on XS4 described by a correlation coefficient $\langle x y\rangle /\left\langle x^{2}\right\rangle=\left|\eta_{y, X S 4} / \eta_{x, X S 4}\right| \simeq 0.85$ which correspond to a tilt angle of $\sim 41^{\circ}$ (here we neglect effects coming from the betatron function contribution).
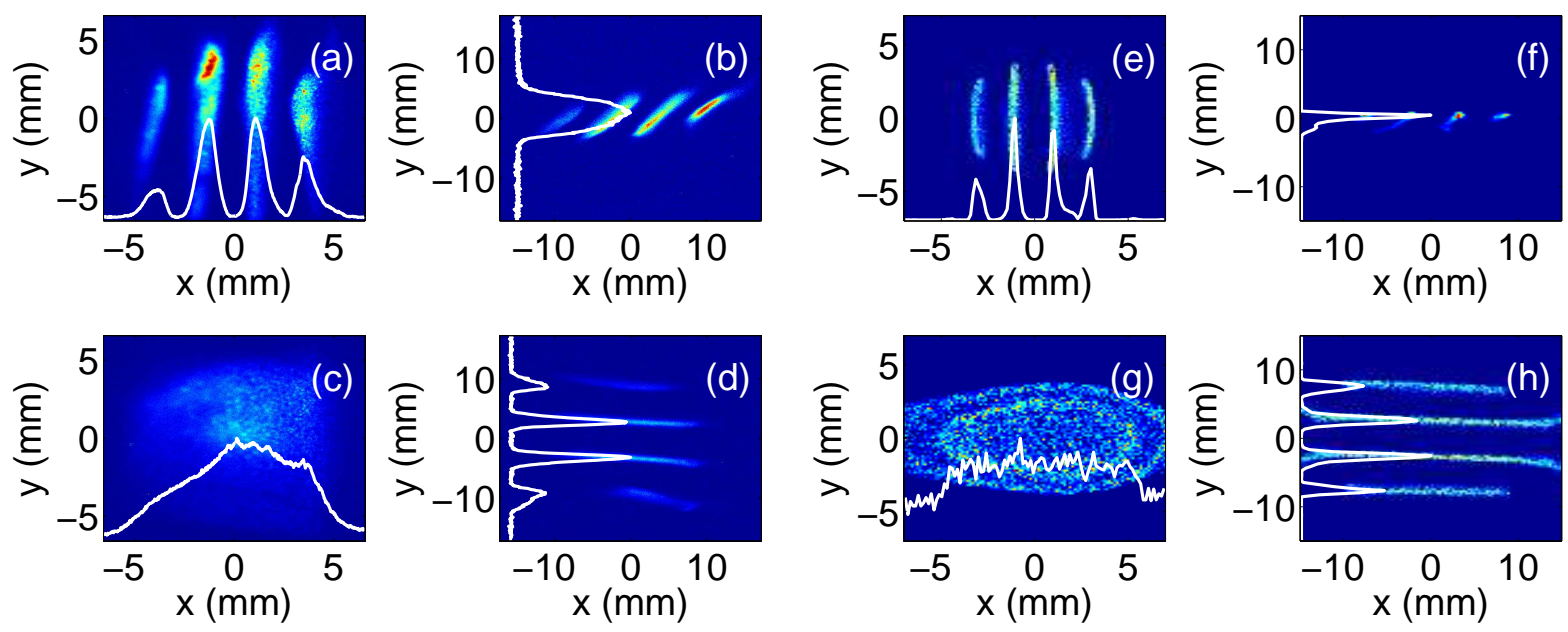

FIGURE 2. Characterization of the transversely modulated beam downstream of the emittance exchanger showing the beam transverse density at X24 [images (a) and (c)] and XS4 [images (b) and (d)] with the deflecting cavity off [images (a) and (b) respectively] and on [images (c) and (d) respectively]. At XS4 [images (b) and (d)] the vertical axis is representative of beam's fractional momentum spread. The plots (e), (f), (g), and (h) are single-particle dynamics simulations performed with ELEGANT corresponding to plots (a), (b), (c), (d). For X24 only the horizontal projections are shown (as white traces) while for XS4 the vertical projections are displayed.

\section{Temporal Modulations}

To characterize the expected temporal modulations we detect and analyze the CTR shining out of a single-crystal quartz window as the beam impinges an aluminum mirror at X24 as schematized in Fig. 3. The spectral fluence radiated 
by a bunch of $N$ electrons by the CTR process is related to the single electron fluence $\left.\frac{d^{2} W}{d \Omega d \omega}\right|_{1}$ via

$$
\left.\frac{d^{2} W}{d \Omega d \omega}\right|_{N}=\left.\frac{d^{2} W}{d \Omega d \omega}\right|_{1}\left[N+N^{2}|S(\omega)|^{2}\right]
$$

where $S(\omega)$ is the intensity-normalized Fourier transform of the normalized charge distribution $S(t)$ [18]. The CTR occurs at frequencies smaller than the cut off frequency $f_{c} \equiv c /\left\{2 \pi \sigma_{z}\left[1+\left[\sigma_{\perp} /\left(\gamma \sigma_{z}\right)\right]^{2}\right]^{1 / 2}\right\}$ where $\sigma_{z}$ and $\sigma_{\perp}$ are respectively the bunch length and transverse size at the CTR radiator location (the beam is assumed to be cylindrically symmetric). In addition if the bunch consists of a train of identical microbunches with periodicity $\tau$ the spectrum will be enhanced at frequencies $f_{n}=n / \tau$ ( $n$ is an integer). The CTR spectrum is representative of the temporal bunch distribution provided $\sigma_{\perp} \ll \gamma \sigma_{z}$. In the present experiment the beam was focused to an rms spot size of $\sigma_{\perp} \simeq 400 \mu \mathrm{m}$ at X24. In addition imperfections due to the frequency-dependent transmissions of the THz beamline components alter the spectrum of the detected CTR. In the present setup only frequencies within the bandwidth $\sim[0.1,3] \mathrm{THz}$ are transmitted. The upper and lower boundaries are respectively set by the cut-off frequency of crystal-quartz window and diffraction effects. The finite bandwidth of the transmission line was taken into account and the expected autocorrelation function is shown in Fig. 4 (a) and (b) for a bunch train consisting of four Gaussian microbunches.
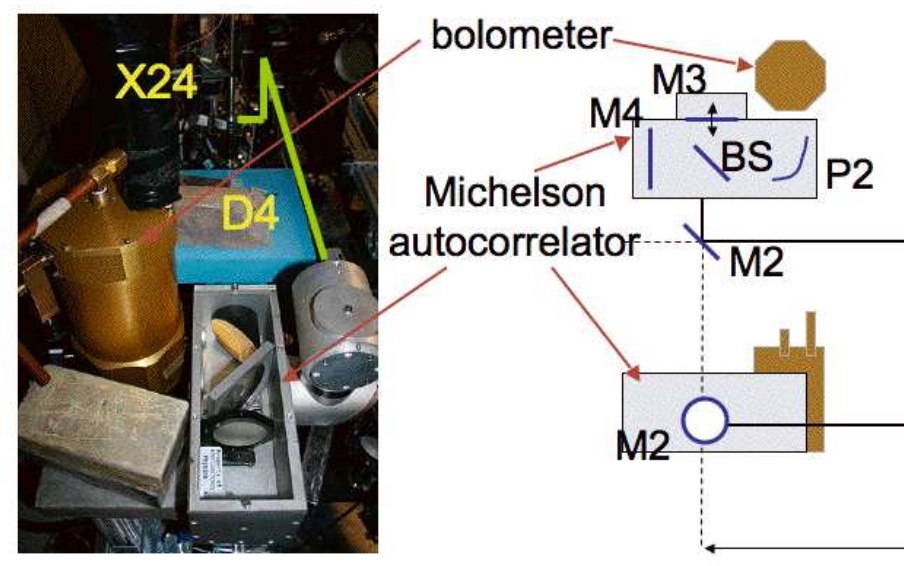

\section{top view}

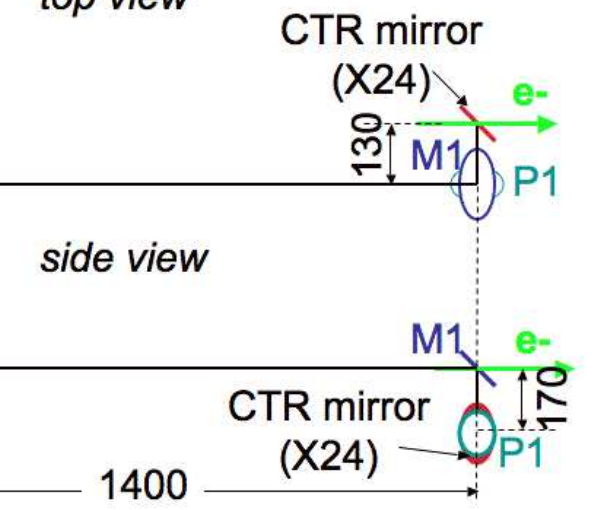

FIGURE 3. Experimental setup for diagnosis of the bunch's temporal distribution via detection of CTR. The radiation is collimated using an off-axis parabolic mirror (P2) and sent to a Michelson autocorrelator (moving mirror is M3). The autocorrelated signal is detected with an He-cooled bolometer. The photo shows the experimental setup (the green lines indicate the path of the CTR). The distances are in $\mathrm{mm}$.

Information on the bunch temporal distribution can be obtained by measuring the total CTR energy collected by the bolometer

$$
\mathscr{W}=\left.2 \pi \int_{\omega_{l}}^{\omega_{u}} d \omega \int_{0}^{\theta_{\max }} d \theta \frac{d^{2} W}{d \Omega d \omega}\right|_{N}
$$

where $\theta_{\max }$ is the angular acceptance of the detection system, $\omega_{l}=0.1 \mathrm{THz}$, and $\omega_{u}=3 \mathrm{THz}$. The signal increases as the bunch duration $\sigma_{t} \equiv \sigma_{z} / c$ decreases. In the limit $\omega_{l} \ll \sigma_{t}^{-1} \ll \omega_{u}$ the radiated energy is inversely proportional to the bunch duration. The final longitudinal C-S parameters downstream of the EEX beamline can be varied by altering the initial horizontal C-S parameters using the quadrupoles $Q_{1}$ and $Q_{2}$. A typical set of measurement obtained by detection of the CTR signal at X24 is shown in Fig. 4 for the cases with (c) and without (d) intercepting the beam with the X3 mutlislit mask. The CTR signal is about two order of magnitudes smaller when the slits are inserted (Fig. 4 (d) is recorded with 50 bunches in the macropulse). The insertion of the multislit mask results in the appearance of a region of coherence for $Q_{1}>1 \mathrm{~A}$. The autocorrelation recorded for the quadrupole settings $\left(Q_{1}, Q_{2}\right)=(1 \mathrm{~A},-1 \mathrm{~A})$ is shown in Fig. 4 (e). When the multislit mask is inserted the autocorrelation is multipeaked. In the present case the peak separation is $\Delta z=390 \pm 20 \mu \mathrm{m}$. Measuring autocorrelation for various quadrupole settings yield different peak separations.

As a final remark, we note that the measurement of beamlets spacing in energy $\Delta \delta$ and along the longitudinal coordinate $\Delta z$ provides the chirp along the bunch $\mathscr{C} \equiv\langle z \delta\rangle /\left\langle z^{2}\right\rangle \simeq \Delta \delta / \Delta z$ where the notation $\left\langle a^{2}\right\rangle$ stands for the 

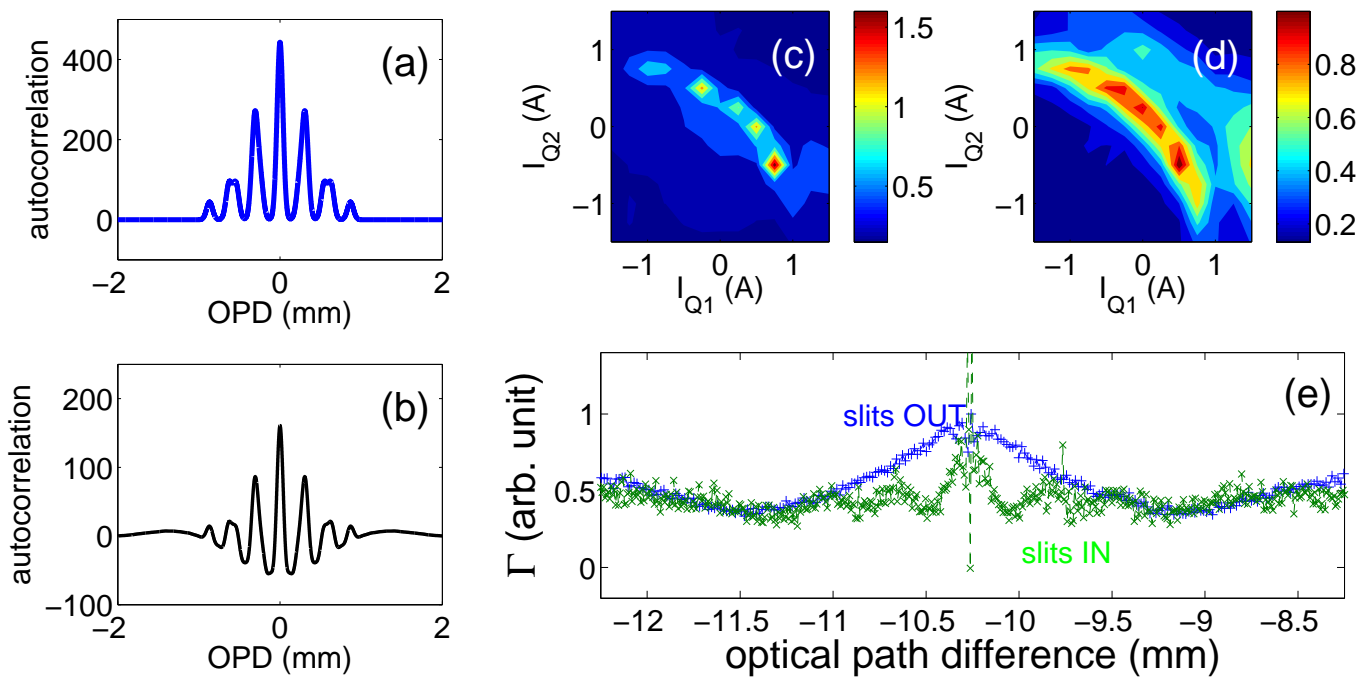

FIGURE 4. Characterization of the temporal bunch distribution downstream of the EEX beamline. Plots (a) and (b) respectively shows the simulated autocorrelation for a train of four Gaussian bunches assuming an ideal THz beamline (a) and a beamline that transmits CTR within frequencies $f \in[0.1,3] \mathrm{THz}$ (the transverse rms spot size is taken to $\sigma_{\perp}=400 \mu \mathrm{m}$ ). The contour plots (c) and (d) correspond to the CTR energy (in arbitrary unit) measured at X24 as a function of quadrupoles $Q_{1}$ and $Q_{2}$ settings without (c) and with (d) intercepting the beam with X3 multislit mask. The measured autocorrelation function $\Gamma(\delta t)$ is displayed in plot (e) without (blue trace) and with (green trace) inserting X3 multislit mask.

centered second-order moment of the variable $a$. The correlation can then be used to infer the longitudinal emittance via

$$
\varepsilon_{z} \simeq \gamma \sqrt{\left\langle z^{2}\right\rangle\left[\left\langle\delta^{2}\right\rangle-\mathscr{C}^{2}\left\langle z^{2}\right\rangle\right]}
$$

where $\gamma$ is the beam's Lorentz factor. We plan to explore this technique for measuring the longitudinal emittance downstream of the EEX beamline. If successful, the would enable the characterization of the final beam emittance over a wide range of incoming beam parameters. Currently, the longitudinal emittance is measured as the product $\sqrt{\left\langle z^{2}\right\rangle\left\langle\delta^{2}\right\rangle}$ and therefore relies on the generation of an upright longitudinal phase space $(\mathscr{C}=0)$ downstream of the EEX beamline. Such a requirement constraints the set of incoming horizontal C-S parameters to a small parameter space thereby preventing parametric studies of the emittance exchange process.

\section{SUMMARY}

We introduced a general method for generating relativistic electron beams with arbitrary current profiles. The proposed method offers a very generic tool for precisely controlling the current distribution (and possibly the emittance partitions) to match the requirements imposed by the front end application. The technique takes advantage of the recently proposed transverse-to-longitudinal phase space exchange to map the horizontal profile associated to the electron bunch into its current profile. In principle any current profile can be produced by properly manipulating the bunch's transverse density to provide the desired horizontal profile upstream of the EEX beamline. We have experimentally demonstrated the ability of the method to produce a train of sub-picosecond electron bunches. The main drawback of shaping the beam using an EEX beamline is the jitter transfer from the longitudinal to transverse plane as pointed out in Ref. [7]. This could be a significant problem for a number of applications, e.g., such as freeelectron lasers. 


\section{ACKNOWLEDGMENTS}

We are indebted to R. Montiel, W. Muranyi, J. Santucci, C. Tan, and B. Tennis for their excellent operational and technical supports. We thank M. Church, H. Edwards, E. Harms and V. Shiltsev for their interest and encouragement. This work was supported by the US Department of Energy under Contract No. DE-FG02-08ER41532 with Northern

Illinois University and by the Fermi Research Alliance, LLC under Contract No. DE-AC02-07CH11359 with the U.S. Department of Energy.

\section{REFERENCES}

1. A. Gover, Phys. Rev. ST Accel. Beams 8, 030701 (2005).

2. C. Jing, A. Kanareykin, J. G. Power, M. Conde, Z. Yusof, P. Schoessow, and W. Gai, Phys. Rev. Lett. 98, 144801 (2007).

3. P Muggli, V Yakimenko, M Babzien, E Kallos, K P Kusche, Phys. Rev. Lett. 101, 054801 (2008).

4. P. Piot, Y.-E Sun, and M. Rihaoui, Proceedings of the 13th Advanced Accelerator Concept workshop (AAC08), AIP Conf. Proc. 1086, 677 (2009).

5. Y.-E Sun, and P. Piot, Proceedings of the 2008 International Linac Conference (LINAC08), Victoria BC, 498 (2009).

6. M. Cornachia, and P. Emma, Phys. Rev. ST Accel. Beams 6030702 (2003).

7. P. Emma, Z. Huang, K.-J. Kim, and P. Piot, Phys. Rev. ST Accel. Beams 9, 100702 (2006).

8. K.-J. Kim and A. Sessler, Proceedings of the 2006 Electron Cooling Workshop, Galena IL (ECOOL06), AIP Conf. Proc. 821, 115 (2006).

9. Y.-E Sun, et al, Proceedings of the 2007 Particle Accelerator Conference, Albuquerque NM (PAC07), 3441 (2007).

10. A.S. Johnson, H.T. Edwards, T.W. Koeth, A.H. Lumpkin, P. Piot, J. Ruan, J.K. Santucci, Y.-E. Sun, R. Thurman-Keup, Proceedings of the 2010 International Particle Accelerator Conference, Kyoto Japan (IPAC10), 4614 (2010).

11. J. Ruan. et al., presented at the 14th Advanced Accelerator Concept workshop, Annapolis, MD, June 13-19, 2010 (these proceedings).

12. P. Piot, et al., submitted to Phys. Rev. ST Accel. Beams (2010); see electronic preprint arXiv:1007.4499v1 (2010).

13. J.-P. Carneiro, et al., Phys. Rev. ST Accel. Beams 8, 040101 (2005).

14. T. Koeth, et al, Proceedings of the 2009 Particle Accelerator Conference, Vancouver BC (PAC09), FR5PFP020 (2009).

15. T. W. Koeth, et al, ibid [9], 3663 (2007).

16. K. Flöttmann, ASTRA: A space charge algorithm, User's Manual, available at http:Www.desy.de/ mpyflo/Astra_Dokumentation

17. M. Borland, "elegant: A Flexible SDDS-Compliant Code for Accelerator Simulation", Advanced Photon Source LS-287, September 2000 (unpublished).

18. J. S. Nodvick and D. S. Saxon, Phys. Rev. 96, 180 (1954). 